\title{
Comparison of Frequency Transform Methods for Condition Monitoring of a DC-Link Capacitor Abdurrahman Raqib ${ }^{1 *}$, Hayder Jahanger ${ }^{1}$, Mark Sumner ${ }^{1}$, Tom Cox ${ }^{1}$, Christian Klumpner', Imran Agha ${ }^{2}$, Richard Kenney ${ }^{2}$, Fernando Aguilar ${ }^{2}$, Peter Lonsdale ${ }^{2}$
}

\author{
${ }^{1}$ Power Electronics, Machines and Control Group, Nottingham, UK \\ ${ }^{2}$ Siemens PLC, Congleton, UK \\ *abdurrahman.raqib@nottingham.ac.uk
}

Keywords: CONDITION MONITORING, CAPACITOR, DEGRADATION, WAVELET, THERMAL

\begin{abstract}
Electrolytic capacitors continue to be used for the DC-link in motor drive systems due to their high specific capacitance. The degradation of electrolytic capacitors can lead to increased equivalent series resistances (ESR) and reduction in capacitance. This can impair the drive behaviour (e.g., reducing the ability of the motor-drive to maintain a steady voltage and thereby maintain steady speed set-points), but will also lead to additional heating and a further cycle of degradation until failure occurs. On-line condition monitoring is useful to understand and predict failure, and can potentially be embedded into a more holistic condition monitoring regime through, for example, the Internet of Things (IoT). It is possible to measure and track currents and voltages through the DC-link and estimate in real-time the corresponding capacitor impedances. The method proposed in this paper will estimate the impedance in a frequency related domain to ensure that the proposed approach is robust to changes in operating conditions - load variation, ambient temperature, drive temperature - and also allow the impedance to be estimated over a wide frequency range. Therefore, different transform methods have been investigated: the Fast Fourier Transform (FFT); the Short-Term Fourier Transform (STFT); and the Continous Wavelet Transform (CWT), making use of two different mother wavelets (complex and bump). It was found that the CWT provided optimal results with fine granularity in time, although selection of a mother wavelet may lead to loss of salient information.
\end{abstract}

\section{Introduction}

Aluminium electrolytic capacitors are commonly used, and are commercially available in cylindrical can form. Commercially produced capacitors are not ideal components, which is to say that the exact value of capacitance varies by batch and by production process, and there is inductance and resistance in each component. These parasitic inductances and resistances are referred to as "Equivalent Series" values.

An issue with these components is degradation over the lifetime of the component. In the case of aluminium electrolytic capacitors, aging of the capacitor causes an increase in the ESR and a variation in the effective capacitance of the component [1]. Depending on the load cycle imposed upon the component as well as the operating environment and circuit application, degradation of the capacitor may not ever become apparent. However, in safety and time critical situations, such as factories employing motor-drives for production purposes, it may be necessary to know when ESR has reached an unacceptable level in order to predicate a maintenance check-up of the motor-drive.

For example, making use of datasheet values and multipliers [2] [3], it is sometimes possible to produce nominal estimates of ESR of a capacitor, assuming specific operating temperatures and ripple currents. However, this is nominal estimate is only suitable as a rule of thumb, as it does not take into account the effect of degradation which accrues as a capacitor ages. It is therefore necessary to provide an estimate of ESR based upon actual capacitor behaviour, instead of an estimate based upon nominal behaviour. Estimates may be found through reading in and using frequency transforms on electrical currents and voltages.

Much work is present in the literature on the issue of estimates and evaluation of the health of an electrolytic capacitor. For example, changes in capacitance and ESR may be observed through the use of scaning electron-microscopy, looking at changes in the foil and oxide layers within capacitor cans [4]. However, this is a destructive method, and it is often better to know the status of health of a capacitor in operation. In addition, analyses based on explicit knowledge of the electrolyte are difficult due to the dearth of information on types of electrolyte used in capacitors [5][6]. There exist online methods of estimating capacitor health in literature, which include proposals which may require extra circuitry to be included in an existing converter [7]; may be application specific and require knowledge of transistor switching states and timings [8]; may require trial and error to find optimal tuning factors [9]; or may simply only work during turn-on and turn-off of power to a power converter, which may not be suitable depending on application [10]. This paper shall look at using mathematical transforms in order to extract information on the state of DC-link capacitors.

\section{Choice of Transforms}

The Fast Fourier Transform (FFT) was initially chosen for this work in order to inspect the frequency response of a motor- 
drive DC-Link across a chosen frequency range of $[300 \mathrm{~Hz}$, $1800 \mathrm{~Hz}$ ]. The FFT is subject to choice of window and window length, where a higher resolution spectrogram may be obtained through a longer sampling period [11]. The implication is that the FFT may produce a coarser resolution output where the sampling period is small. This situation may matter in cases where motor-drive output transients occur quickly and the sampling period is long enough that it includes the transient and produces an erroneous output calculation.

The Discrete Fourier Transform, from which the FFT is derived and implemented, is described in (1):

$$
X(k)=\sum_{n=0}^{N-1} x(n) \cdot e^{\frac{-j 2 \pi k n}{N}} \text { where } k=0,1,2 \ldots N-1
$$

In order to have gain a better snapshot of very rapid transients, the Short Term Fourier Transform (STFT) was employed. The frequency resolution of the output of the STFT is dependent on window length as well as choice of the window function [12], where a well-chosen window length may provide a wealth of information about the frequencies of interest in a given time-window. However, the implication then is that an optimal window length would need to be decided, where the chosen window would have to be able to deal with electrical signals that may vary massively depending on the motor load torque profile. It is understood that the STFT may be beneficial for cases where monitoring of steady-state signals may not provide enough information about intermittent loweramplitude, higher frequency signals, where such signals may be indicative of deviation from long-term steady-state behaviour, i.e. degradation.

The discrete STFT is described in (2):

$$
X(n, \omega)=\sum_{m=-\infty}^{\infty} x(m) \cdot w(n-m)^{-j \omega n}
$$

Both the FFT and STFT are referred to as frequency transforms, where each transform is provided with timevarying data; a snapshot of the data is taken and analysis is performed; and a frequency-vs-amplitude response is returned, where frequency is the independent variable and amplitude is the dependent variable. Related to frequency transforms are time-frequency transforms, where the response is in the form of time-vs-frequency-vs-amplitude responses: in this case, time is the independent variable, and frequency and amplitude the dependent variables - i.e. that changes in amplitude and frequency may be observed with change in time, as opposed to the FFT, where one output result is the nominally the same for all moments of time in the input signal. An example of a timefrequency transform is the Continuous Wavelet Transform (CWT) [13], which finds itself suited to motor-drive applications where variation is to be expected in electrical signals due to change in the mechanical load torque profile, i.e. where steady-state behaviour in the electrical signals is periodically irregular.

Wavelet transforms rely upon choice of mother wavelets, which are mathematical functions that are used to split the input signal into smaller signals of different frequencies and amplitudes [14]. The choice of mother wavelet is a restriction in a similar vein to the window of the STFT, where an improperly chosen mother wavelet may lead to imprecise output from the transform, which would then affect analysis and decision-making further down the stream. Furthermore, there are any number of ways to express a mother wavelet mathematically, causing more issues in choosing an exact mother wavelet. However, it is fortuitous that there are a number of predefined mother wavelets in the literature from which to choose, due in part to the popularity of the wavelet transform for frequency analysis [15]. These mother wavelets are available in pre-written library forms in different languages and for different platforms, such as Python or MATLAB [16] [17].

The general CWT is described in (3), where $\boldsymbol{s}$ is scale and $\boldsymbol{p}$ is position:

$$
w(s, p)=\int_{-\infty}^{\infty} f(t) \cdot \varphi(s, p, t) \cdot d t
$$

(3) states that the output response of the CWT is the sum over time of the input signal scaled and shifted versions of the mother wavelet. The presence of a shift implies that the mother wavelet must have a centre frequency and room within which to shift, i.e. a bandwidth. Similarly, the presence of a scaling factor implies that the mother wavelet must change in size at different frequencies, increasing or decreasing as the frequency changes compared to the centre frequency.

For example, the frequency responses of specific frequencies were found using the complex Morlet wavelet through the implementation of (4), where $\boldsymbol{f}_{\boldsymbol{c}}$ is the cut-off frequency of choice for the mother wavelet - in this case, $2 \mathrm{~Hz} ; \boldsymbol{f}_{\boldsymbol{s}}$ is the sampling frequency - in this case, $1 \mathrm{MHz}$; and $\boldsymbol{a}$ is the scaling factor used to find the frequency $\boldsymbol{f}$ of interest. The bandwidth of the mother wavelet was chosen to be $10 \mathrm{~Hz}$ in this case.

$$
f=\frac{f_{c} f_{s}}{a}
$$

After some preliminary testing with different wavelets and simple test-cases using the MATLAB platform, two specific mother wavelets were chosen: the complex Morlet wavelet [18] and the bump wavelet [19]. The complex Morlet wavelet was well received in literature for impedance estimation, and the bump wavelet was a mother wavelet from a pre-written toolbox which was used to compare performance against the complex Morlet wavelet.

For the choice of type of window, a Blackman window was found to suffice; there was no noticeable difference that was 
observed between uses of Blackman or Hamming windows in this work.

\section{Simulation of Electrical Models}

A simple approach when taken when looking for an estimate of the ESR through capturing and processing electrical signals: voltage across and current through a capacitor was recorded, and a measure of the ESR was extracted.

To begin, the model of a capacitor was specified as shown in Fig. 1. This model involved a capacitor being held in parallel with a very large leakage resistance, and in series with an ESR. The equivalent series inductance (ESL) was neglected as the effective ESL impedance would be very small in the frequency range of interest, assuming an ESL in the low nano-Henries.

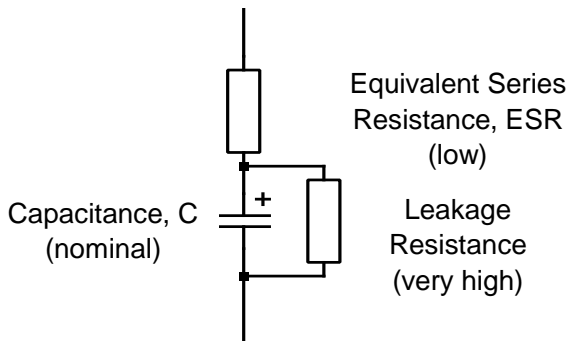

Fig. 1 - Capacitor model

Next, the outline of a typical motor-drive was designed and simulated using the PLECS power electronics simulation package [20]. The circuit simulation is shown in Fig. 2. This motor-drive was designed to be powered by a $415 \mathrm{~V}, 50 \mathrm{~Hz}, 3 \mathrm{ph}$ source with a very small mains impedance and a very small cable impedance; PWM frequency was chosen to be $4 \mathrm{kHz}$. High-frequency bypass capacitors were placed in parallel with a network of DC-link capacitors and bleed resistors, where the resistors were included to speed up capacitor discharge upon the removal of a power supply. The high-frequency bypass capacitors were low enough in capacitance to not overly affect the nominal capacitances of the DC-link capacitors. Values are listed in Table 1.

Table 1 - Simulated circuit values

\begin{tabular}{cccc}
\hline Parameter & Value & Parameter & Value \\
R6 & $10 \mu$ & R19 & $330 \mathrm{k}$ \\
L4 & $10 \mu$ & C4 & $270 \mu$ \\
C10 & $100 \mathrm{n}$ & C5 & $270 \mu$ \\
C11 & $22 \mathrm{n}$ & R1 & ESR \\
C12 & $22 \mathrm{n}$ & R7 & $100 \mathrm{k}$ \\
R16 & $330 \mathrm{k}$ & R11 & ESR \\
R17 & $330 \mathrm{k}$ & R12 & $100 \mathrm{k}$ \\
R18 & $330 \mathrm{k}$ & & \\
\hline
\end{tabular}

The output of the drive was connected to a $2.2 \mathrm{~kW}$ induction motor with a controllable load torque profile, where the induction motor was vector-controlled. An RC low-pass filter of $10 \mathrm{kHz}$ was included in the simulation in order to filter recorded data, and a zero-order hold of $10 \mu \mathrm{s}$ was used for sampling in order to mitigate the effect of aliasing [21].
The motor load torque profile was varied to provide a $2 \mathrm{~Hz}$ periodic load to the motor. The values of the capacitors $\mathrm{C} 4$ and $\mathrm{C} 5$ in Fig. 2 were each held at $270 \mu \mathrm{F}$ for a total capacitance of $135 \mu \mathrm{F}$, and simulations were run in the cases where R1 was equivalent to R11, where both were set at values of $0.5^{*}[1 \mathrm{~m} \Omega$, $10 \mathrm{~m} \Omega, 100 \mathrm{~m} \Omega, 500 \mathrm{~m} \Omega, 1 \Omega]$. The motor load torque profile was varied between no load and rated load: five sets of recordings were made of voltage across and current through the DC-link capacitors. The periodic signal is shown in Fig. 3.

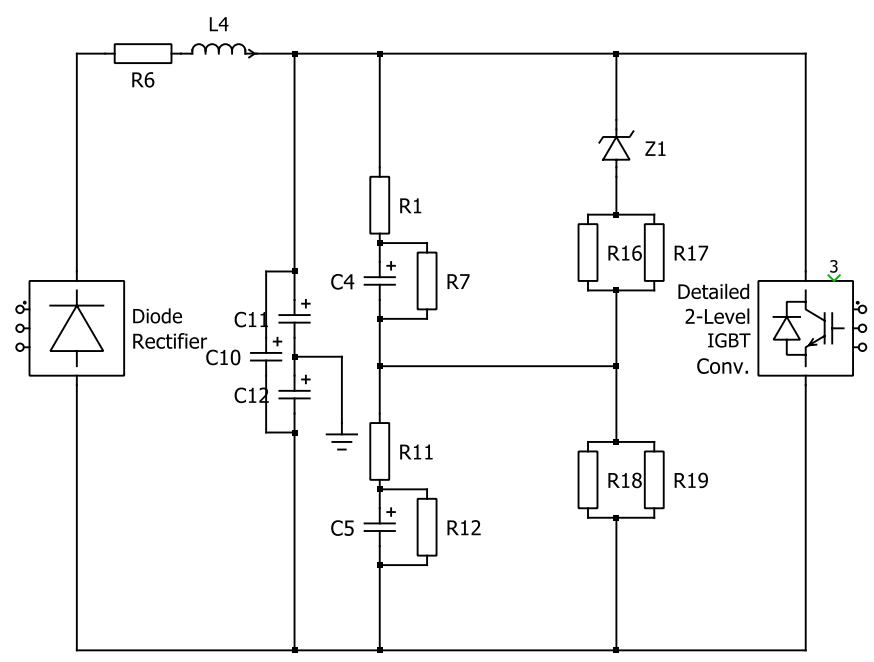

Fig. 2 - Simulated circuit

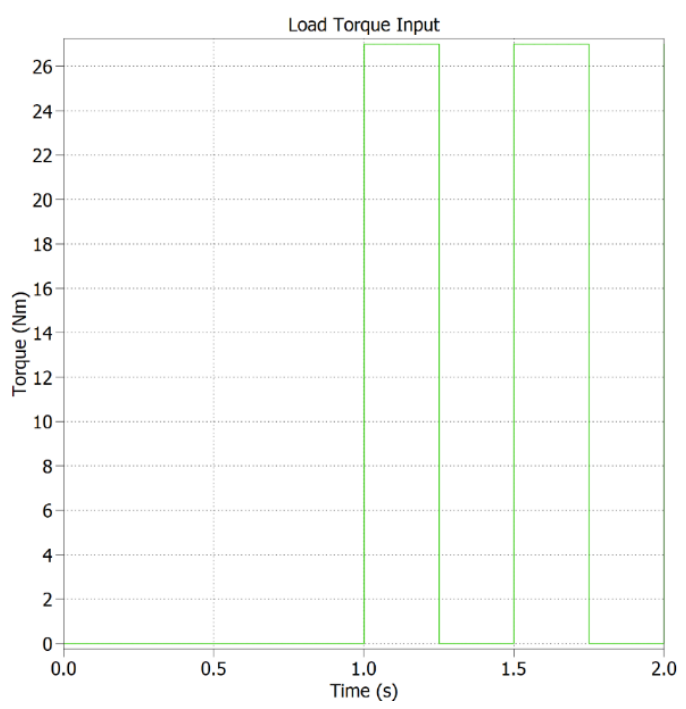

Fig. 3 - Load torque input

Voltage and current were filtered and sampled at $1 \mathrm{MHz}$ and saved to a data file over the duration of one second when the load torque was changing, as shown in Fig. 3.

The MATLAB platform was used to import and process data in order to find the DC-link impedances for each of the five simulations, all of which were subject to a varied mechanical load. Making use of known frequency transform relations, the input voltage and current data were used to find impedance as shown in (5), where $F$ represents each of the three different transforms considered [22]. 


$$
F(z(t))=\frac{F(v(t))}{F(i(t))}
$$

Scripts were written to import one second of data from the PLECS simulation and perform calculations upon the data. For each value of ESR, the different transforms were used to provide estimates of capacitance and ESR at the frequencies of $[300 \mathrm{~Hz}, 600 \mathrm{~Hz}, 900 \mathrm{~Hz}, 1200 \mathrm{~Hz}, 1500 \mathrm{~Hz}, 1800 \mathrm{~Hz}]$.

Regression analysis was then used to find influential outliers through measuring abnormally large Cook's distances, where frequencies associated with abnormally large Cook's distances were neglected in the next step [23]. Cook's distances can be used to detect values in a set of data that are dissimilar to the other values, and are useful when attempting to perform a regression analysis on data that has data-points that are not representative of the entire data as a whole: in this case, it is assumed that abnormal values would show up in estimates due to measurement noise and quantisation issues [21], which was considered to be an acceptable assumption.

A mean was taken of the resultant values in order to provide a mean wideband estimate. Across a range of values of ESR, the results of the estimation schemes are shown in Fig. 4, and Fig. 5 shows the absolute percentages of error associated with the estimates.

In the case of estimation of capacitance where the output motor was subject to a varied mechanical load torque profile, it was found that the FFT and STFT produced estimates that were very close to the true value of capacitance, with a very low absolute error between set and estimated values. This was also the case for the bump wavelet, which had been chosen as a test wavelet against which to compare the complex Morlet wavelet. In fact, it was found that the complex Morlet wavelet produced estimates which increased in error as the ESR increased, as opposed to the other transforms which demonstrated a convergence in estimate as ESR increased. This divergence may be explained by the fact that the complex Morlet was found to suffer from a phase-shift issue, where the presence of an increasing real component in impedance causes a pronounced error during estimation.

In the case of the estimation of ESR where the output motor was subject to a varied mechanical load torque profile, it was found that the four transforms listed quickly converged to the true value of ESR as ESR increased, but STFT gave the most accurate results, within a $10 \%$ error band. It was noted that the bump wavelet and the FFT had comparable results, and the complex Morlet wavelet converged slowly to the true value of ESR. It is interesting to note that the complex Morlet wavelet diverged in its estimate of capacitance as ESR increased, but converged in its estimate of ESR as ESR increased. This follows on from the assumption of a phase-shift issue during estimation, where a real element would dominate the imaginary element of the impedance, and estimation would be skewed towards the real element as the ESR increases. Investigation of this phase-shift issue has not yet been conclusive.
Overall, it was understood that the complex Morlet wavelet was generally unsuitable for a DC-link impedance estimation scheme compared to the other three transforms, judging by simulation observations. Additionally, the STFT was discounted due to the issues involved in deciding an optimal window length that may suit multiple real-world applications, where transients may be unpredictable. Choosing between the FFT and bump wavelet, both are understood to be suitable for estimation of capacitance and ESR, but it is probable that having knowledge of estimated capacitance and ESR variation with finer granularity in time may be useful for diagnostic and maintenance purposes, and therefore the bump wavelet may be chosen as the optimal in this situation for monitoring the condition of a DC-link.

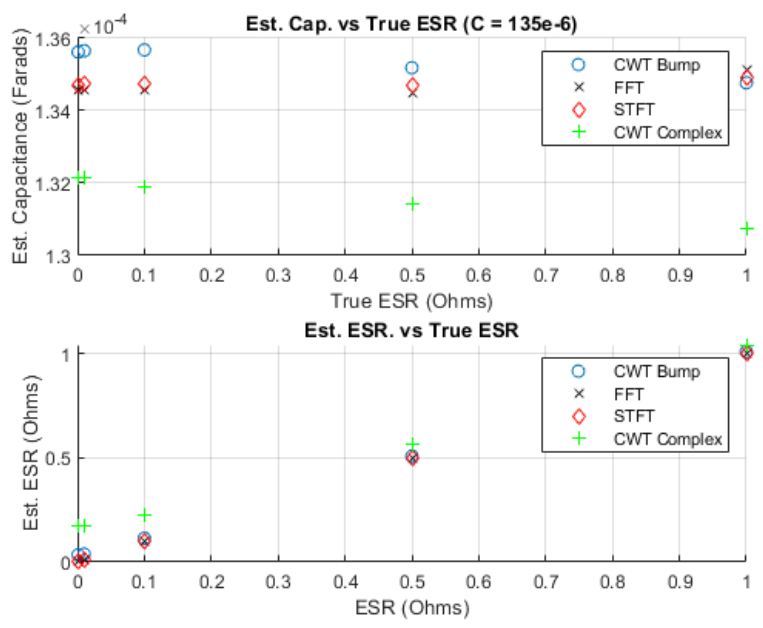

Fig. 4 - ESR estimation results
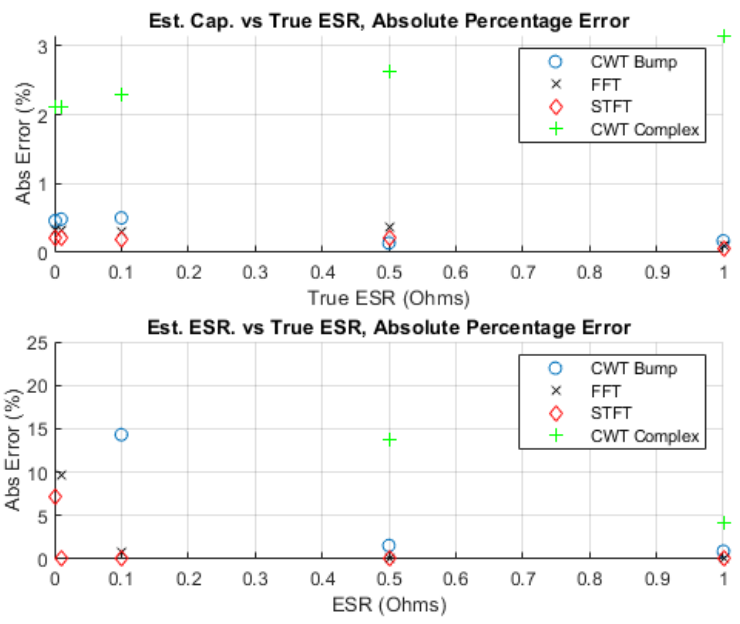

Fig. 5 - Capacitance estimation results

\section{Thermal Issues}

As noted in [5], a well tested thermal model may help verify estimates of ESR found through use of an estimation scheme based on electrical signals.

The capacitor can may be considered as a thermal network, as shown in Fig. 6, where the capacitor case is represented as a 
first-order RC network [24]. The internal hotspot of the capacitor, which may be considered as the central capacitor core, would be losing power in the form of heat due to imperfections in the manufacturing process, as well as due to the presence of resistive elements internal to the device. This power loss would bleed from the capacitor core to the capacitor case, and thereafter to the ambient environment.

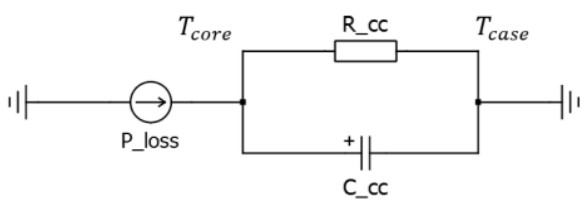

Fig. 6 - First-order thermal model

(6) may be used to explain Fig. 6:

$$
T_{\text {core }}-T_{\text {case }}=P_{\text {loss }} \cdot R_{c c} \cdot\left(1-e^{\frac{-t}{R_{c c} \cdot C_{c c}}}\right)
$$

Depending on the information provided by manufacturers, it may be possible to build thermal models as a starting point of understanding thermal behaviour of capacitor cans. To demonstrate, consider the values in Table 2.

Table 2 - Operating conditions

\begin{tabular}{ccccc}
\hline $\begin{array}{l}\text { Vdc } \\
(\mathrm{V})\end{array}$ & $\begin{array}{c}\text { Irms } \\
(\mathrm{A})\end{array}$ & $\begin{array}{c}\text { Frequency } \\
(\mathbf{H z})\end{array}$ & $\begin{array}{c}\text { Thermal } \\
\text { Resistance } \\
\text { (core to } \\
\text { can) } \\
(\mathbf{K} / \mathbf{W})\end{array}$ & $\begin{array}{c}\text { ESR at } \\
\mathbf{3 0 0 H z} \\
\mathbf{6 0} \mathbf{C} \\
(\mathbf{m} \Omega)\end{array}$ \\
10 & 0.44 & 300 & 1.3 & 120 \\
\hline
\end{tabular}

Assuming a capacitor is held at 10VDC and has a $300 \mathrm{~Hz}$ 0.44Arms ripple current injected into it, at fixed ambient conditions, temperatures associated with the body of the capacitor can will be higher than the surrounding ambient environment. In this case, the manufacturer states that the thermal resistance between the core and the can has a value of $1.3 \mathrm{~K} / \mathrm{W}$

Assuming the current and ambient temperature are held constant, and that the can and core temperatures listed are at steady state, it is possible to extract a few variables of interest from this set of data.

Given a known power loss and a fixed ripple current, (7) may be used directly to find a resistance analogous to the ESR of the capacitor:

$$
R_{\text {loss }}=\frac{P_{\text {loss }}}{I_{\text {ripple }}^{2}}
$$

This output value may be used to compare against the estimated ESR found through the use of the transforms discussed above. However, this method relies upon a knowledge of the power loss value associated with the capacitor.

One straightforward method of estimating the power loss value would be to subject the capacitor to steps in temperature through the use of a heating chamber. The capacitor would be required to have thermocouples placed around the exterior, and recordings of temperature would be taken from the moment of step in temperature from a lower to a higher value. Through analysis of the temperature rise curves; through comparisons of expected ESR vs estimated power loss, for calibration purposes; or through batch testing of heating capacitors, it may be possible to extract a good estimate of power loss for a given capacitor, so as to better inform the estimate of ESR provided by electrical-based estimation.

Making use of TDK B43547 capacitors produced with Type$\mathrm{K}$ thermocouples embedded within the can, it was possible to produce an estimate of ESR based upon temperature. Table 3 shows results based upon three such capacitors used.

Table 3 - Estimated ESR and errors

\begin{tabular}{ccc}
\hline Capacitor & $\begin{array}{c}\text { Estimated } \\
\text { ESR } \\
(\mathbf{\Omega})\end{array}$ & $\begin{array}{c}\text { Absolute } \\
\text { Error } \\
(\boldsymbol{\%})\end{array}$ \\
1 & 0.149 & 29 \\
2 & 0.149 & 29 \\
3 & 0.020 & 100 \\
\hline
\end{tabular}

It was understood that estimation error was introduced due to making use of an imperfect heating chamber, where the lossy chamber allowed heat to leak easily, leading to inaccuracy in measuring and maintaining the ambient temperature, and - by extension - the temperature of the can. In the case of the third capacitor, the test was repeated twice in the exact conditions of the first two capacitors, but the error was consistently high: it is understood that this may be due to the internal placement and position of the thermocouple, where the third can would have had enough of a slight difference compared to the first two capacitors that the measured internal temperature would then drastically affect any calculations based upon (6).

\section{Conclusion}

Three types of frequency transforms were compared and evaluated for the purpose of estimating DC-link capacitance and ESR in a simulated motor-drive, based on electrical waveforms. The CWT was found to be optimal for observing changes in estimate of capacitance and ESR with finer granularity in time, which would be desirable for observing changes in time for product health-management and maintenance reasons. Thermal issues were also noted, where estimation based on electrical signals may be compared with estimation based on thermal signals for verification purposes; problems were observed about the issue of estimating power loss in thermal models, where power loss varies with operating conditions. Further work must be undertaken to analyse the identified thermal issue. 


\section{Acknowledgements}

This work was funded through a CASE award with Siemens PLC - Standard Drives, Congleton, and supported by the Engineering and Physical Sciences Research Council.

\section{References}

[1] BS60384: 'Fixed capacitors for use in electronic equipment', 2009

[2] TDK EPCOS, 'Aluminium electrolytic capacitors: Snap-in capacitors: B43547' (TDK EPCOS, 2016)

[3] TDK EPCOS, 'Frequency and temperature multipliers: B43547' (TDK EPCOS, 2016)

[4] Shrivastava, A.: 'Reliability Evaluation of Liquid and Polymer Aluminum Electrolytic Capacitors'. PhD thesis, University of Maryland, 2014

[5] Gasperi, M.L.: 'Life prediction model for aluminum electrolytic capacitors', IEEE Industry Applications Conference, San Diego, USA, 1996

[6] Gasperi, M.L.: 'A method for predicting the expected life of bus capacitors', IEEE Industry Applications Conference, New Orleans, USA, 1997

[7] Venet, P., Perisse, F., El-Husseini, M.H., et al: 'Realization of a smart electrolytic capacitor circuit', IEEE Industry Applications Magazine, 2002, 8, (1), pp. 16-20

[8] Lee, K., Kim, M., Yoon, J., et al: 'Condition Monitoring of DC-Link Electrolytic Capacitors in AdjustableSpeed Drives', IEEE Transactions on Industry Applications, 2008, 44, (5), pp. 1606-1613

[9] Lee, D., Lee, K., Seok, J., et al: 'Online capacitance estimation of DC-link electrolytic capacitors for three-phase AC/DC/AC PWM converters using recursive least squares method', IEE Proceedings - Electric Power Applications, 2005, 152, (6), pp. 1503-1508

[10] Li., H., Xiang, D., Han, X., et al: 'High-Accuracy Capacitance Monitoring of DC-Link Capacitor in VSI Systems by LC Resonance', IEEE Transactions on Power Electronics, 2019, 34, (12), pp. 12200-12211

[11] Dorf, R., Bishop, R.: 'Modern Control Systems' (Pearson Prentice Hall, 2008)

[12] Portnoff, M.: 'Time-frequency representation of digital signals and systems based on short-time Fourier analysis', IEEE Transactions on Acoustics, Speech, and Signal Processing, 1980, 28, (1), pp. 55-69

[13] Abusorrah, A.: 'The Application of the Continuous Wavelet Transform to Power System Identification and Protection'. PhD thesis, University of Nottingham, 2006

[14] Tavner, P., Ran, L., Penman, J., et al: 'Condition Monitoring of Rotating Electrical Machines' (The Institution of Engineering and Technology, 2008)

[15] Rana, K., Thakur, S.: 'Comparisons of wavelets and algorithms based on wavelets and comparing the results with JPEG', International Conference on Energy, Communication, Data Analytics and Soft Computing, Chennai, India, 2017

[16] 'Continuous Wavelet Transform (CWT)', https://pywavelets.readthedocs.io/en/latest/ref/cwt.html, accessed November 2019
[17] 'Wavelet Toolbox', https://uk.mathworks.com/products/wavelet.html, accessed November 2019

[18] Jia, K.: 'Impedance Based Fault Location in Power Distribution Systems'. PhD thesis, University of Nottingham, 2012

[19] 'Bump Wavelet', https://uk.mathworks.com/help/wavelet/ref/cwtft.html\#buu64 ch, accessed November 2019

[20] 'PLECS Standalone',

https://www.plexim.com/products/plecs_standalone, accessed November 2019

[21] Franklin, G., Powell, J., Workman, M.: 'Digital Control of Dynamic Systems' (Addison-Wesley Publishing Company, 1980)

[22] Liu, B., Jia, K.: 'Comparison of system impedance estimating methods', International Conference on Advanced Power System Automation and Protection, Beijing, China, 2011

[23] Duivesteijn, W., Feelders, A., Knobbe, A.: 'Different Slopes for Different Folks: Mining For Exceptional Regression Models with Cook's Distance', ACM SIGKDD International Conference on Knowledge Discovery and Data Mining, Beijing, China, 2012

[24] Prasanth, S., Sathik, M., Sasongko, F., et al: 'Condition Monitoring of Electrolytic Capacitor based on ESR Estimation and Thermal Impedance model using Improved Power Loss Computation', International Power Electronics Conference, Niigata, Japan, 2018 